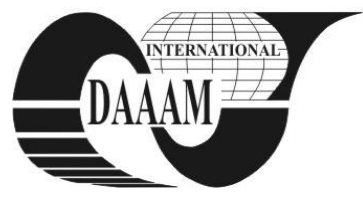

\title{
EXPERIMENTAL CONTROL SYSTEM FOR MOBILE ROBOT WITH ULTRASONIC SENSORS
}

\author{
SHIPOVALOV, E[gor] \& PRYANICHNIKOV, V[alentin] E.
}

\begin{abstract}
Experimental control system for caterpillar mobile robot featuring ultrasonic sensors with analog echo signal processing is described. The need for ultrasonic-based navigation is explained as well as challenges associated with it. A simple analog circuit for ultrasonic echo signal input using consumer PC sound adapter is described.
\end{abstract}

Key words: ultrasonic sensor, navigation of mobile robots, spike elimination, range finder

\section{INTRODUCTION}

The task of inspecting dangerous areas that cannot be manned for prolonged periods of time or at all is becoming increasingly more common. For example, it often arises during liquidation of disasters involving hazardous materials or high levels of radiation.

Remotely controlled mobile robots (MR) that are typically used in these circumstances have a serious drawback: they completely depend on having a constant data connection to the operator. Should it be lost or interrupted due to, for example, radiation-induced radio wave blockage, they stop operating. This dictates the need for MR that is able to navigate without such a permanent connection (Grishin, 1989).

The need for ultrasonic sensors in such a system follows from the limitations of sensors of other types. Video-based sensor systems are much less reliable and require optics which can lose transparency when subjected to aerosol drops or high levels of radiation. Laser-based systems are relativelyt expensive and have high power consumption which reduces the maximum time of autonomous operation of the MR (Grishin \& Zhmylevskaya, 1991).

The range of tasks to be performed in dangerous areas can be quite wide and include creating a map, collection of samples, navigating to a point with certain coordinates or characteristics - like hotbed of fire or radiation. These tasks may require special algorithms for controlling the MR, but these would be built using the same basic functions that are implemented in this work.

\section{CHALLENGES ASSOCIATED WITH USING ULTRASONIC SENSORS FOR NAVIGATION}

Most ultrasonic range finders work by measuring the time it takes emitted signal to travel to the reflecting surface and back. The distance can then be calculated using the following formula: $d=c * t / 2$, where $t$ is the aforementioned time and $c$ - the speed of sound which is $343 \mathrm{~m} / \mathrm{s}$ under standard atmospheric pressure and temperature of $20 \mathrm{C}$.

Implementation of this technique has its challenges. Detection of echo signal can sometimes be difficult due to interference of waves on different paths and because of spatial characteristics of reflecting surface. The less even is the surface and the higher is its angle relative to direction of signal emission, the more of echo signal is dispersed and ends up undetected by the sensor. In some industrial environments, background noise can be a problem, like one generated by pneumatics (Pryanichnikov, 1981) Even under favourable conditions achievable resolutions are limited by wave length and angle of beam, the latter being about 30 degrees (Kondratyev et al, 1983).

\section{HARDWARE USED}

In this work, Polaroid-6500 $49 \mathrm{kHz}$ ultrasonic sensor taken from a household range finder is utilised. The following circuitry has been developed for filtering and amplifying the echo signal to enable its digitisation:

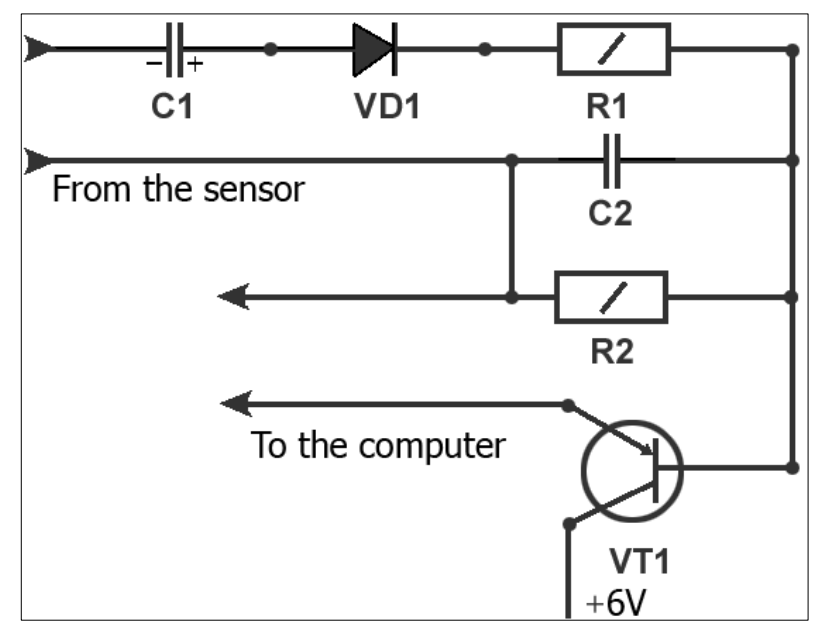

Fig. 1. Ultrasonic transducer interface circuit. Elements used: $C 1-10 \mathrm{mcF}, \mathrm{C} 2-180 \mathrm{pF}, \mathrm{Rl}-1 \mathrm{KOm}, \mathrm{Rl}-1 \mathrm{KOm}, \mathrm{VD} 1-$ D511, VT1-2SC633

There are two reasons for this. One is that ultrasonic signal lies outside frequency range of Analogue to Digital Converters used in PC sound input circuits. This is worked around with an RC-circuit based on $0.1 \mathrm{mcF}$ capacitor. Another is the need to amplify and compress echo signal. If this is not done, automatic input level adjustment system in PC audio input chain reacts to emitted signal and makes the echo signal indistinguishable from background noise. This is solved by bringing the amplifying transistor into state of saturation.

\section{SOFTWARE AND ALGORITHMS}

To make the software maintainable and easier to test and develop, it was divided into three modules: Data Collection module, Decision Making module, and Control module. Each of these modules was developed largely independently of the others. 


\subsection{Data collection module}

Data collection module initiates the ultrasonic transducer, reads digitised echo signal, filters out noise and calculates measured distance for passing on to decision making module. The transducer is triggered via low-current electromagnetic relay connected to parallel port of the PC. The relay operates with low enough currents to prevent damaging the port circuitry. Duration of impulse is $0.1 \mathrm{~s}$. Immediately after the impulse is emitted echo signal is read for the period of one second. It is then processed and analysed to arrive at a measurement.

Optimal sampling frequency for thSis sensor system has been experimentally determined as $11 \mathrm{KHz}$. Therefore, onesecond long sample is encoded in $44 \mathrm{~KB}$ of data. The following is an illustration of a sample. The interval delimited with vertical lines depicts "window" of spike elimination algorithm:

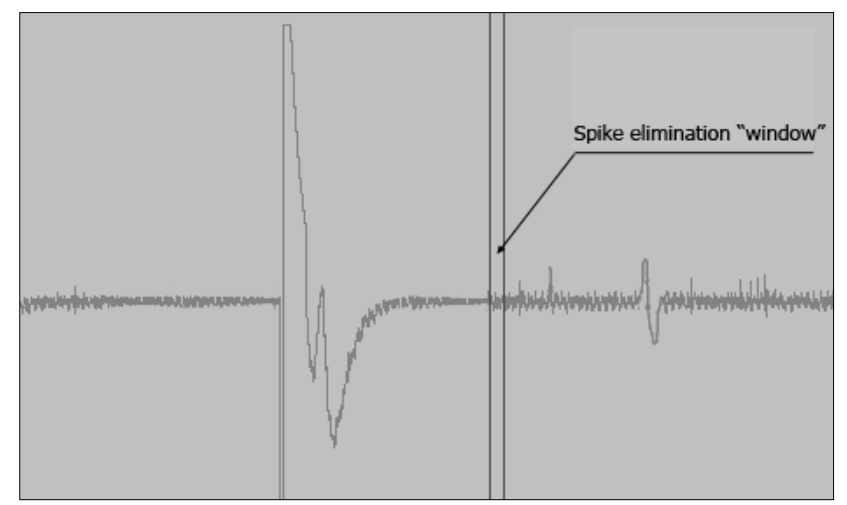

Fig. 2. Example of digitised echo signal with spike elimination "window"

\subsection{Decision making module}

Decision making module regularly queries data collection module, analyses the data it returns and if necessary alters the course of moving MR by sending commands to control module. This work includes implementation of basic functions required for crossing land with undetermined surface configuration at a speed of up to $1 \mathrm{~m} / \mathrm{s}$. This includes obstacle avoidance and recovering from dead ends. Obstacle avoidance algorithm can be formulated as the following set of rules:

1. If neither of the two sensors detects obstacles, move towards sub goal.

2. If the right sensor detects an obstacle, turn left until rule 1 comes into effect. Same for left sensor and right turn.

3. If both sensors detect obstacles, turn right until either rule 1 or 2 comes into effect. After each obstacle avoidance manoeuvre, sub goal is recalculated.

The control program retains path that MR has followed so far using odometer readings. When a sub goal is detected to be already on that path the direction of turn in rule 3 is reversed. This method, despite its simplicity, allows recovering from most dead ends (Petrina \& Popov, 1992), (Kirilchenko, 1980)

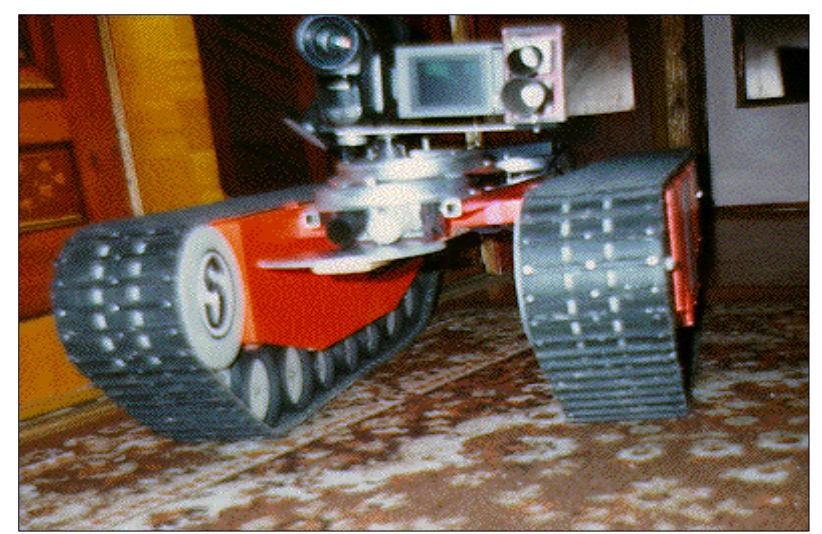

Fig. 3. Mobile robot platform used in this work

\subsection{Control module}

The control module provides network command interface to the mobile platform and allows controlling the robot via mnemonic commands. For example: "SET_CATERP_SPEED 110 " sets speed of caterpillar \#1 to value of 10, "SCAN" returns distance to the obstacle (if any), "ODOMETER" returns odometer reading. These commands are converted into byte codes that are transmitted over serial interface to microcontrollers of the mobile platform.

It is implemented as a background daemon program in Linux environment. This architecture has been chosen due to its flexibility and author's extensive professional experience implementing client-server software. Any client, local or remote, can interface with MR's control module as long as it supports the TCP network protocol. Other options under consideration included popular remote access protocols such as REST or SOAP-based web services, and CORBA, but were ruled out as too complicated and not meeting the requirements of low traffic and low latency. Although controlling MR via web interface over a wireless channel can be a useful option.

\section{CONCLUSION}

This work yielded a number of valuable practical results that get us closer to building an adequate general-purpose MR. The three-tier application architecture (data collection, decision making and control modules) proved itself as the right approach to modularising MR software. Field tests have shown adequacy of ultrasonic sensors for basic collision avoidance on a relatively high-speed caterpillar-driven MR. Feasibility of processing of analogue ultrasonic echo signal using accessible off-the-shelf components has been experimentally proven.

\section{FUTURE PLANS}

The authors plan to continue refining the software to make measuring distance to uneven surfaces more reliable. There are plans to use newer transducers with narrower beams as well as transducer arrays to allow for simultaneous location and mapping (SLAM).

\section{REFERENCES}

Grishin B.A. (1989). Mobile robots used in extreme conditions. Analytical review of machine building and robotics, VNII TEMR, Moscow, USSR, p. 82. (Russian)

Grishin M.M., Zhmylevskaya N.S (1991), Mobile and moving robots used in non-manufacturing fields, VNII TEMR, Moscow, Russia, 278 p. (Russian)

Kirilchenko A.A. (1980). Development of algorithms and software system for processing of range measurement data and route planning of mobile robots, Candidate thesis, specialty code 01.01.10, Keldysh Institute of Applied Mathematics, Moscow, USSR, 184 p (Russian)

Kondratyev Y.A., Koltsov V.N., Pryanichnikov V.E (et al), (1983), Development of acoustic locator for autonomous mobile robots, Robotics and automatisation of manufacturing processes. Barnaul, USSR, AMTSN-TI, Part 1, p 186-187 (Russian)

Pryanichnikov V.E. (1981). Modelling of controlled mobile robot with acoustic sensor, Candidate thesis, specialty code 01.02.01, defended on 20.01.1981 at special council D002.40.02 at Zhukovsky VVIA, Moscow, USSR, 1981, 192 p., (Russian)

Petrina N.A., Popov E.P. (1992), Expert systems in robotics, VINITI, Moscow, Russia, 181 p (Russian) 